\title{
An open-source implementation of estimation and correction of head-motion and eddy-current distortions generalizable across diffusion MRI signal models
}

\author{
D. Pisner ${ }^{1}$, C. Provins ${ }^{2}$, S. Tourbier ${ }^{2}$, G. Lerma-Usabiaga ${ }^{3}$, A. Rokem ${ }^{4}$, and O. Esteban ${ }^{2}$. \\ ${ }^{1}$ Department of Psychology, University of Texas at Austin, TX, USA; ${ }^{2}$ Dep. of Radiology, Lausanne University \\ Hospital and University of Lausanne, ${ }^{3}$ BCBL. Basque Center on Brain, Cognition and Language, ${ }^{4}$ Department of \\ Psychology and eScience Institute, The University of Washington
}

Synopsis (100 words): We develop an open-source tool for the retrospective estimation of inter-volume head-motion and eddy-current distortions, typically found in diffusion MRI (dMRI) data acquired with echo-planar imaging schemes. The implementation is "open-since-inception" to ensure transparency. By leveraging the widely used DIPY package and a user-friendly interface, researchers have at their disposal an implementation combining state-of-art approaches with substantial improvements that can efficiently leverage any compliant diffusion model(s) while simultaneously accounting for susceptibility distortions.

Summary (250 char): We release an open-source software for dMRI eddy-current and head-motion corrections. This implementation generalizes on eddy, SHOREline, and DIPY, now taking multiple acquisition schemas. Validated using unit tests and FiberFox phantom simulations.

\section{$\operatorname{Body}(850$ words $)$}

Introduction: Retrospective estimation of head-motion between diffusion-weighted images (DWI) acquired within diffusion MRI (dMRI) experiments renders exceptionally challenging ${ }^{1}$ for datasets including high-diffusivity (or "high b") images. These "high b" $\left(b>1000 \mathrm{~s} / \mathrm{mm}^{2}\right)$ DWIs enable higher angular resolution, as compared to more traditional diffusion tensor imaging (DTI) schemes. UNDISTORT ${ }^{1}$ (Using NonDistorted Images to Simulate a Template Of the Registration Target) was the earliest method addressing this issue, by simulating a target DW image without motion or distortion from a DTI $\left(b=1000 \mathrm{~s} / \mathrm{mm}^{2}\right)$ scan of the same subject. Later, Andersson and Sotiropoulos ${ }^{2}$ proposed a similar approach (widely available within the FSL eddy tool), by predicting the target DW image to be registered from the remainder of the dMRI dataset and modeled with a Gaussian process. Besides the need for less data, eddy has the advantage of implicitly modeling distortions due to eddy currents. More recently, Cieslak et al. ${ }^{3}$ integrated both approaches in SHORELine, by (i) setting up a leave-one-out prediction framework as in eddy; and (ii) replacing eddy's general-purpose Gaussian process prediction with the SHORE ${ }^{4}$ diffusion model. Here, we propose an open implementation of eddy-current and head-motion correction that builds upon the work of eddy and SHORELine, while generalizing these methods to multiple acquisition schemes (single-shell, multi-shell, and diffusion spectrum imaging) using diffusion models available with DIPY ${ }^{5}$.

Methods: To allow dMRI researchers flexibility, generality, and ease of use, we propose a modular implementation as follows:

1) Data representation: dMRI datasets (and especially, "high b") are typically large, as they contain a 3D map of many diffusion directions. Consequently, the memory requirements of algorithms quickly become prohibitive. Our implementation reduces this burden by importing the original DWIs and all the necessary acquisition parameters (e.g., b-values and b-vectors) into an HDF5 object. HDF5 is a scientific data format that allows memory-mapped access to data (i.e., only the sections of the dataset that will be used at a given time are actually copied from a file system into memory). The data representation object has also built in the leave-one-volume out splitting strategy for convenient access at each iteration of the estimator (step 3).

2) Model factory: a proxy class provides a simplified interface to the data model that will be used to predict the registration target. This proxy class follows closely the high-level interface of DIPY for their diffusion models, although it also allows other modeling options (such as a Gaussian process, or a naive model that returns a $b=0$ map for testing purposes). 
3) Estimator: orchestrates the appropriate model selected by the user. It leverages the data representation object to generate DWI registration input (each DWI) and target (as generated by the model), and calls the ANTs registration tool $^{6}$ for the estimation of the head motion parameters and the linear transformation parameters induced by Eddy currents.

We evaluate our tool with two strategies: 1) we validate the software implementation of the three major components with comprehensive unit-tests; and 2) we execute our tool using the simplest DTI model on four in-silico, realistic phantoms, generated with FiberFox ${ }^{7}$, with low- and high- motion and with-/without- eddy current simulation (and combinations thereof).

Results: We openly release a software package under an Apache 2.0 license that transparently builds upon two successful methodologies - eddy and SHORELine - in pursuit of an extensible framework for next-generation diffusion MRI preprocessing. The tool overcomes certain limitations of FSL's eddy, namely the noncommercial user license, the restriction to "shelled" data, and the limitation to the Gaussian process for data prediction; and of SHORELine, with an optimized, generalizable interface that is not limited to just the SHORE model. We further evaluate that the major modules described above indeed perform as expected with unit tests (e.g., by generating a ground-truth dataset realigning the same DWI that has been simulated with known head-motion and eddy-current distortions). Finally, we validate that the tool also provides reasonable results based on realistic phantom data including established simulation benchmarks generated from the ISMRM 2015 tractography challenge ${ }^{8}$.

Discussion and conclusion: The dMRI eddy-current and head-motion correction tool we present here is a new transparent and open-source implementation based on state-of-the-art algorithms. It is also an important building block of dMRIPrep-- an up-and-coming preprocessing pipeline for dMRI inspired by the wide adoption of fMRIPrep ${ }^{9}$. Through this new community-driven module, we were able to develop an open architecture that can easily and flexibly draw upon diverse diffusion models while also generalizing to a variety of data acquisition schemes. This module, as with all modules in dMRIPrep, equips dMRI researchers with a reliable and transparent tool that is efficient, easy to use and maintain, public-interest steering and creates new opportunities for improving data quality and the reproducibility of dMRI experiments. 


\section{VSM}

A voxel-shift map may be set to account for susceptibility distortions

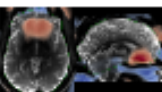

\section{Transforms}

Affine matrices

corresponding to the DWls

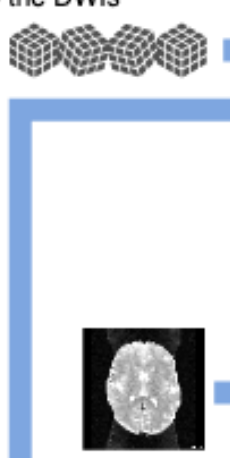

$b=0$ map

Reference map obtained from

"low-b" or b $\sim 0$ volumes

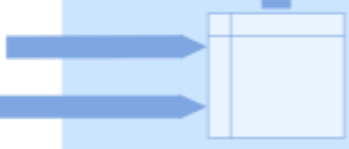

DWIs

Diffusion-weighted images, which do

not include "low-b" (b 0) volumes.
Estimator

Orchestrates dMRI representation, model, and alignment, iterating until finished.
The VSM is projected to each of the DWIs through the corresponding affine, producing distortioncorrected DWIs

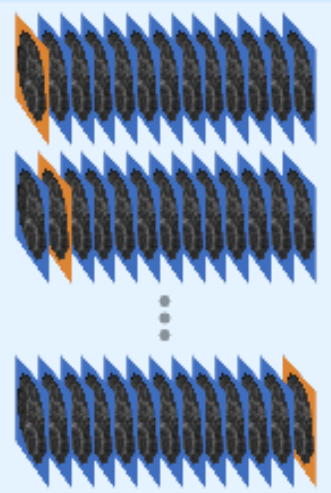

\section{Data splitter}

Separates data into train and target DWI, leaving one DWI out each iteration.

dMRI representation

Image-to-image alignment

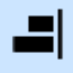

\section{Target DWI}

One DWI that will be aligned to the synthetic counterpart.

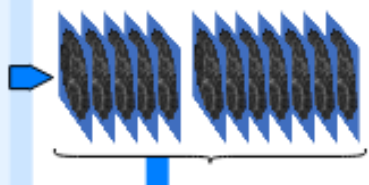

Train DWI

The remainder of the

DWIs is fit a model.

Data modeling \& Prediction

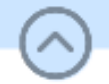
DIPY 


\section{References:}

1. Ben-Amitay, S., Jones, D. K. \& Assaf, Y. Motion correction and registration of high b-value diffusion weighted images. Magn. Reson. Med. 67, 1694-1702 (2012).

2. Andersson, J. L. R. \& Sotiropoulos, S. N. An integrated approach to correction for off-resonance effects and subject movement in diffusion MR imaging. NeuroImage 125, 1063-1078 (2016).

3. Cieslak, M. et al. QSIPrep: an integrative platform for preprocessing and reconstructing diffusion MRI data. Nat. Methods 18, 775-778 (2021).

4. Ozarslan, E., Koay, C. G., Shepherd, T. M., Blackband, S. J. \& Basser, P. J. Simple Harmonic Oscillator Based Reconstruction and Estimation for Three-Dimensional Q-Space MRI. in Proc. Intl. Soc. Mag. Reson. Med. vol. 171396 (2009).

5. Garyfallidis, E. et al. Dipy, a library for the analysis of diffusion MRI data. Front. Neuroinformatics 8, 8 (2014).

6. Avants, B. B. et al. A reproducible evaluation of ANTs similarity metric performance in brain image registration. NeuroImage 54, 2033-44 (2011).

7. Neher, P. F., Laun, F. B., Stieltjes, B. \& Maier-Hein, K. H. Fiberfox: An Extensible System for Generating Realistic White Matter Software Phantoms. in Medical Image Computing and Computer-Assisted Intervention (eds. Schultz, T., Nedjati-Gilani, G., Venkataraman, A., O’Donnell, L. \& Panagiotaki, E.) 105-113 (2013). doi:10.1007/978-3-319-02475-2_10.

8. Maier-Hein, K. H. et al. The challenge of mapping the human connectome based on diffusion tractography. Nat. Commun. 8, 1349 (2017).

9. Esteban, O. et al. fMRIPrep: a robust preprocessing pipeline for functional MRI. Nat. Methods 16, 111-116 (2019). 